\title{
Psychometric Testing of the Korean Version of the Caring Behaviors Inventory-24 in Clinical Nurses
}

\author{
Kang, Younhee ${ }^{1) \oplus} \cdot$ Kang, Sook Jung ${ }^{2) \oplus} \cdot$ Yang, In-Suk ${ }^{3) \odot} \cdot$ Lee, Haeok $^{4) \oplus} \cdot$ Fitzpatrick, Joyce $^{5)}$
}

1) Professor, College of Nursing, Ewha Womans University, Seoul 2) Assistant Professor, College of Nursing, Ewha Womans University, Seoul 3) Associate Professor, Department of Nursing, Kyungil University, Gyeongsan, Korea 4) Professor, College of Nursing and Health Sciences, University of Massachusetts, Boston, MA 5) Professor, Frances Payne Bolton School of Nursing, Case Western Reserve University, Cleveland, OH, U.S.A

\begin{abstract}
Purpose: To determine psychometric properties of the Caring Behaviors Inventory-24 (CBI-24) among Korean clinical nurses. Methods: A methodological design was used. Data were collected from 408 clinical nurses. Construct validity analysis was performed, including factorial, convergent, and discriminant validity. Internal consistency was tested by Cronbach's $\alpha$ coefficients, inter-item correlation, and corrected item-total correlation. Results: Exploratory factor analysis produced three factors: 'empathy and supporting', 'knowledge and skills', and 'providing comfort'. In confirmatory factor analysis results, model fit indices were acceptable $\left(x^{2} / \mathrm{df}=3.50\right.$, RMR $=.05$, RMSEA $=.08$, $\mathrm{CFI}=.90$ ). The values obtained for the AVE ranged from .53 to .68 , and for the CR ranged from .53 to .68 . Convergent validity coefficients were noticeably greater in magnitude than discriminant validity coefficients: .53 ( $A V E_{1}$ value) and .68 ( $\mathrm{AVE}_{2}$ value $) \geq .45\left(\mathrm{r}_{12}{ }^{2}\right.$ value $)$ and $.68\left(\mathrm{AVE}_{2}\right.$ value $)$ and $.63\left(\mathrm{AVE}_{3}\right.$ value $) \geq .61\left(\mathrm{r}_{23}{ }^{2}\right.$ value). Internal consistency (Cronbach's $\alpha$ ) of CBI-K was .95. Conclusion: The CBI-K was shown to have acceptable construct validity and good internal consistency. Study findings imply that $\mathrm{CBI}-\mathrm{K}$ could be a useful instrument for clinical administrators and nursing researchers to assess caring behaviors among Korean clinical nurses. Utilization of $\mathrm{CBI}-\mathrm{K}$ might contribute to the building of empirical knowledge and the understanding of caring behaviors from nurses' perspectives.
\end{abstract}

Key Words: Behavior; Caring; Nurses; Nursing; Psychometrics

Received Aug 16, 2020 Revised Dec 14, 2020 Accepted Feb 3, 2021

Corresponding author: Yang, In-Suk https://orcid.org/0000-0002-9599-2721

Department of Nursing, Kyungil University

50 Gamasil-gil, Hayang-up, Gyeongsan 38428, Korea

Tel: +82-53-600-5664, Fax: +82-53-600-5679, E-mail: insookyang@gmail.com 


\section{INTRODUCTION}

Caring is posited as one of the core concepts within the meta-paradigm of nursing science. Caring is also considered as a broad and complex concept. It is believed to have philosophical ethical-ontological subjective attributes [1]. Whether caring can be measured remains controversial. However, empirical evidence is needed to deeply understand the nature of caring [1]. For these reasons, many studies have been conducted to clearly understand the caring behavior that conveys caring [2-6]. Nurses' caring behavior is believed to be able to maintain and promote a patient's physical and mental well-being. It can also improve nursing care quality and patients' satisfaction related to health care $[2,7,8]$.

There is no universal definition of caring, although there is some consensus on ideas and principles related to caring. Several studies have examined caring or caring behavior from nurses' and patients' perspectives [4,6]. In the case of survey studies, most of these studies used instruments based on well-established theoretical frameworks such as Watson's theory of human caring [9] and Swanson's theory of caring [10].

Watson has suggested that caring should be understood with humanistic-aesthetic views and scientific views [11]. She emphasized ontological competencies in nursing science and reported carative factors grounded in a humanistic value system [11]. Watson's original work has evolved and it has been redefined as caritas processes [11]. In Latin, caritas refers to "caring as something precious and fragile and that must be sustained"[12]. Caring, that is caritas in nursing science, refers to interactions and relationship between patients and nurses. It has been carried out based on humanity, compassion, and love [12]. Swanson has defined that caring is "a nurturing way of relating to a valued other toward whom one feels a personal sense of commitment and responsibility [10]". She has demonstrated five essential processes that characterize caring: knowing, being with, doing for, enabling, and maintaining belief [10]. Finfgeld-Connett [7] has addressed caring in terms of intellectual, moral, and interpersonal components. Caring is considered as a cohesive, context-specific interpersonal process that is represented by expert nursing practice, interpersonal sensitivity, and intimate relationships [7]. As consequences of caring, physical and mental well-being of patients and mental well-being of nurses are enhanced [7].

Caring behavior is a multidimensional concept that has a variety of different meanings and interpretations [13]. Caring behavior could be explained as humanistic, scien- tific, instrumental, and expressive caring. Humanistic caring refers to a practicing of loving, kindness, empathy, and equanimity for self and others, which are universal and altruistic values [11]. Scientific caring means making choices and implementing of acts based on knowledge about the caring process, its purpose, organization and outcome [13]. Instrumental caring means doing something technically, which might be technical competence that is being implemented to meet patients' basic physical needs (e.g., starting an intravenous injection and administering needed analgesics). Expressive caring refers to nursing activities based on established interpersonal relationship. It should reflect the value of each person. It should be imbued with values of respect, dignity, and individuality (e.g., respecting and accommodating privacy needs) $[13,14]$.

Several instruments are available to measure caring behaviors. The Caring Factor Survey [12] evaluates patients' perceptions of caritas caring behaviors received from nurses. Caritas caring behaviors which is a combination of carative factors involves the integration of transpersonal caring and love within a caring moment of the nurses-patients relationship. The Caring Assessment Report Evaluation Q-Sort (Care-Q) [15] is the most frequently used instrument for identifying the most important caring behaviors that are perceived by patients while nurses provide caring to patients. Care-Q was composed of 50 behavioral items, 6 subscales: accessible, comforts, anticipates, develops a trusting relationship, monitors and follows through, and explains and facilitates. These instruments have a limitation in obtaining comprehensive understanding because they contained only a few of the properties of caring behaviors and mainly measured caring behaviors from perspectives of patients.

Caring Behaviors Inventory (CBI) is a tool that includes all attributes of humanistic, scientific, instrumental, and expressive caring. The CBI was developed to understand of nurses' caring behaviors in clinical setting. This inventory was first described by Wolf in 1981 and consisted originally of 75 items [16]. Subsequently, using the responses of 278 nurses and 263 patients, Wolf and colleagues performed a factor analysis and reduced the inventory to 42 items with 5 dimensions: assurance of human presence, positive connectedness, professional knowledge and skill, respectful deference to other, and attentive to other's experience [17]. This 42-item version was modified to a 24-item version with 4 dimensions [6]. This CBI-24 version was shown to have high internal consistency ( $\alpha$ $=.96$ for both patients and nurses) and test-retest reliability ( $\mathrm{r}=.88$ for patients, $\mathrm{r}=.82$ for nurses), as well as convergent validity ( $\mathrm{r}=.62$ for patients) [6]. The CBI-24 is a useful short 
version evaluating caring behaviors, which could measure multidimensional aspects for caring.

Recent studies have been focused on the importance of caring behaviors from not only patients' perspective, but also nurses' perspectives in western cultures [4,18-20]. However, there is a lack of evidence for understanding of caring behaviors perceived by patients and nurses in Korean culture [21,22]. One of the possible reasons is that there is a lack of psychometrically sound instruments in Korea to measure caring behaviors. The lack of instruments that encompasses Korean culture to measure caring behaviors has led to limited quantitative research into health-related outcomes and attributes of caring behaviors. Psychometric testing of the CBI-24 was performed among nursing students and patients $[3,6]$ but homogeneity of participants were not guaranteed. Thus, there is a need to establish an instrument of caring behaviors that could measure nurses' caring behaviors considering Korea culture.

\section{Aim}

The aim of the study was to translate the Caring Behaviors Inventory-24 (CBI-24) into Korean and test psychometric properties of the Korean version of the CBI (CBI-K) for Korean clinical nurses.

\section{METHODS}

\section{Study Design}

A methodological design was used to conduct psychometric analysis.

\section{Instrument}

The questionnaire for the collection of data included demographics and the CBI-24[6]. The CBI constructed by Wolf [16] and Wolf et al.[17] was one of the earliest instruments for measuring caring behaviors. It was developed with a conceptual-theoretical base which considered the caring as an interpersonal relationship that occurred between nurses and patients. In addition, the CBI-24 has been used to determine the perception of the frequency of caring behaviors as practiced by nurses and/or received by patients in hospitals. The advantage of this instrument is that it is simple and easy to administer [1]. Each item in the CBI-24 was evaluated with a 6-point Likert-type scale, with higher mean score indicating higher frequency of caring behaviors. The Cronbach's $\alpha$ of this instrument was .96 in a previous study [6]. Permission to use and translate the CBI was obtained from the original developer of the instrument prior to the study.

\section{Study Procedures}

\section{1) Translation}

The CBI-24 was translated into Korean by standard forward and back translation procedures. The original CBI24 was primarily translated from English into Korean by a bilingual expert on an item-by-item basis. It was then translated back into English by a bilingual expert to check the content-suitability of the translated text to the English text. Two bilingual translators who are familiar with a bicultural background in Korea and U.S. were able to ensure whether the meaning of each item was conceptually and idiomatically appropriate, regarding cultural and contextual characteristics.

\section{2) Content validity testing}

(1) Participants and data collection

The content validity of each item was evaluated by ten nursing professionals with a doctoral degree and clinical experience. Ten experts were asked to rate each item based on relevance: $1=$ not relevant; $2=$ somewhat relevant; $3=$ quite relevant; $4=$ highly relevant [23].

(2) Data analysis and results

Content validity of CBI-24 was calculated by item-level (Item Content Validity Index, I-CVI) and scale-level content validity index using the averaging calculation method (Scale Content Validity Index/Average, S-CVI/Ave). Values of I-CVI and S-CVI/Ave were calculated based on proportion of experts who gave relevance rating 3 or 4 to each item and average of I-CVI for all items of the instrument, respectively [24]. As a result, percent of I-CVI ranged was .80 to 1.00 and percent of S-CVI/Ave was .99 .

\section{3) Construct validity and reliability testing}

(1) Participants and data collection

Data were collected using participant-completed questionnaires from a convenience sample of clinical nurses in two general hospitals in Korea. After explaining the purpose and procedure of this study to the manager of each institution, participants were recruited in clinical settings. Data collection was performed from October 1 to October 20, 2014. Eligibility criteria for participating in this study were as follows: 1 ) Registered Nurses (RNs) aged 19 years or older, 2) with the ability to respond to the questionnaire. A total of 470 questionnaires were distributed to two general hospitals and 408 questionnaires were completed. 
Tabachnick and Fidell [25] have recommended that the sample size for factor analysis should be 300 or more.

(2) Data analysis

For data analysis, only 408 questionnaires were used after removing questionnaires with missing data. Demographic data were analyzed using frequencies and percentage. For psychometric test of the CBI-K, construct validity analysis was performed, including factorial, convergent, discriminant validity, and reliability analyses. All analyses were conducted using SPSS Amos 17.0 and IBM SPSS Statistics Version 26.0.

Factorial validity was first confirmed by Exploratory Factor Analysis (EFA). It was then confirmed by Confirmatory Factor Analysis (CFA). The CFA was performed to assess the goodness of fit of the model, including $x^{2}$, ratio of $x^{2}$ by degrees of freedom $\left(x^{2} / \mathrm{df}\right)$, Root Mean Square Residual (RMR), Root Mean Square Error of Approximation (RMSEA), Goodness of Fit Index (GFI), Adjusted Goodness of Fit Index (AGFI), Normed Fit Index (NFI), Incremental Fit Index (IFI), Turker-Lewis Index (TLI), and Comparative Fit Index (CFI). The fit of the model was considered adequate when $x^{2} / \mathrm{df} \leq 5.0, \mathrm{RMR} \leq .05$, RMSEA $\leq .08$, GFI $\geq .90$, AGFI $\geq .90, \mathrm{NFI} \geq .90$, IFI $\geq .90$, TLI $\geq .90$, and $\mathrm{CFI} \geq .90[26,27]$.

Convergent validity was estimated based on the calculation of the Average Variance Extracted (AVE) and Composite Reliability (CR) proposed by Fornell and Larcker [28]. Convergent validity was used to ensure if items of each factor were strongly interrelated. Values of AVE $\geq .50$ and $C R \geq .70$ were considered adequate.

Discriminant validity was used to assess whether the items that composed one factor were not strongly correlated with another factor. This validity was evaluated through a correlational analysis. It was considered adequate when $A V E_{i}$ and $A V E_{j} \geq r_{i j}^{2}\left(r_{i j}^{2}\right.$ : square of the correlation between factors $i$ and j) [28].

An internal consistency was estimated through Cronbach's $\alpha$ coefficients, inter-item correlation, and corrected item-total correlation. Generally, values of Cronbach's $\alpha$ $\geq .70$, inter-item correlation $\geq .20$, corrected item-total correlation $\geq .30$ are considered as adequate [29].

\section{4) Ethical considerations}

This study was approved by the institutional review board of the affiliated university (IRB No.: 80-4). Participants of this study voluntarily agreed to take part in this study and signed the informed consent form after they were provided with a written explanation of this study.

\section{RESULTS}

\section{Demographic Characteristics of Participants}

Table 1 presents demographic characteristics of the 408 clinical nurses. Their mean age was 27.72 years (Standard Deviation, $[\mathrm{SD}]=4.86$ ), ranging from 22.00 to 51.00 years. The majority $(97.1 \%)$ of participants were women. More than two thirds $(67.9 \%)$ of participants had a bachelor's degree. Most (90.4\%) participants had less than or equal to 10 years of experience in clinical settings. About $60 \%$ of subjects worked in an internal medicine ward and/or an intensive care unit.

Table 1. Demographic Characteristics of Participants $(N=408)$

\begin{tabular}{llc}
\hline Characteristics & Variables & $\mathrm{n}(\%)$ \\
\hline Gender & Women & $396(97.1)$ \\
& Man & $12(2.9)$ \\
Age (year) & $<30$ & $281(68.9)$ \\
& $30 \sim 39$ & $115(28.2)$ \\
& $\geq 40$ & $12(2.9)$ \\
Education & Associate & $117(28.7)$ \\
& Bachelor & $277(67.9)$ \\
& Master & $14(3.4)$ \\
Total experience & $<1$ & $82(20.1)$ \\
(year) & $1 \sim 2$ & $92(22.5)$ \\
& $3 \sim 5$ & $66(16.2)$ \\
& $6 \sim 10$ & $129(31.6)$ \\
& $\geq 11$ & $39(9.6)$ \\
Experience unit & Internal medicine ward & $123(30.1)$ \\
& Surgical ward & $83(20.3)$ \\
& Intensive care unit & $109(26.7)$ \\
& Others & $93(22.8)$ \\
\hline
\end{tabular}

\section{Construct Validity}

\section{1)Factorial Validity}

Prior to performing EFA, the stability of data for factor analysis was assessed. The Kaiser-Meyer-Olkin value was .95 , exceeding the recommended value of 60 [29]. Bartlett's test of sphericity [29] reached statistical significance.

The 24-item CBI was analyzed by EFA (maximum likelihood estimation and oblique rotation). One item (No. 15 - "Treating patient information confidentially") was dropped because it had low factor loading (below .40) for all factors. Twenty-three items and three factors were common to clinical nurses. As a result, 'factor 1 (empathy and supporting)' consisted of fourteen items, 'factor 2 (knowledge and skill)' consisted of four items, and 'factor 
3 (providing comfort)' consisted of five items (Table 2).

The EFA revealed that the CBI-K had three dimensions. The scale was then assessed by CFA, which showed that the three-factor model had the following goodness of fit values: $x^{2}=853.38(p<.001), x^{2} / \mathrm{df}=3.76, \mathrm{RMR}=.05$, RMSEA= $.08, \mathrm{GFI}=.84, \mathrm{AGFI}=.81, \mathrm{NFI}=.86, \mathrm{IFI}=.89$. TLI $=.88$, and $\mathrm{CFI}=.89$ for the three-factor model. We added the error covariance to the three-factor model using modification indices in Amos version 17.0 and the goodness of fit improved to $x^{2}=788.38(p<.001), x^{2} / \mathrm{df}=3.50, \mathrm{RMR}=.05$, $\mathrm{RMSEA}=.08, \mathrm{GFI}=.85, \mathrm{AGFI}=.82, \mathrm{NFI}=.87, \mathrm{IFI}=.90, \mathrm{TLI}=$ .89 , and $\mathrm{CFI}=.90$ as a result. The error covariance correc- tion model was selected. Factor loading values (path coefficients) obtained were statistically significant $(p<.001)$ (Table 2).

\section{2) Convergent Validity}

Convergent validity was adequate for factors "empathy and supporting", "knowledge and skill", and "providing comfort" of the 23-item CBI-K. As shown in Table 3, the AVE values for Factor 1, Factor 2, and Factor 3 were .53, .68 , and .63, respectively. The CR values for each factors were $.94, .89$, and .90 , respectively.

Table 2. Caring Behaviors Inventory Items, Factors, and Descriptive Statistics

$(N=408)$

\begin{tabular}{|c|c|c|c|c|c|c|c|}
\hline \multirow[b]{2}{*}{ No. } & \multirow[b]{2}{*}{ Items } & \multirow[b]{2}{*}{$\mathrm{M} \pm \mathrm{SD}$} & \multirow{2}{*}{$\begin{array}{c}\text { Corrected } \\
\text { item-total } \\
\text { correlations }\end{array}$} & \multicolumn{4}{|c|}{ Factor loading } \\
\hline & & & & $\begin{array}{l}\text { Empathy and } \\
\text { supporting }\end{array}$ & $\begin{array}{l}\text { Knowledge } \\
\text { and skill }\end{array}$ & $\begin{array}{l}\text { Providing } \\
\text { comfort }\end{array}$ & $\beta$ \\
\hline 1. & Attentively listening to the patient & $4.58 \pm 0.74$ & .66 & .66 & & & .68 \\
\hline 2. & Giving instructions or teaching the patient & $4.31 \pm 0.93$ & .56 & .58 & & & .59 \\
\hline 3. & Treating the patient as an individual & $4.55 \pm 0.98$ & .62 & .64 & & & .64 \\
\hline 4. & Spending time with the patient & $3.71 \pm 1.09$ & .55 & .69 & & & .61 \\
\hline 5. & Supporting the patient & $4.23 \pm 0.86$ & .69 & .80 & & & .73 \\
\hline 6. & Being empathetic or identifying with patient & $4.22 \pm 0.86$ & 67 & .78 & & & .73 \\
\hline 7. & Helping the patient grow & $4.03 \pm 0.91$ & .73 & .79 & & & .78 \\
\hline 8. & Being patient or tireless with the patient & $4.04 \pm 0.88$ & 65 & .70 & & & .69 \\
\hline 13. & $\begin{array}{l}\text { Allowing the patient to express feelings about his } \\
\text { or her disease and treatment }\end{array}$ & $4.15 \pm 0.91$ & .70 & .71 & & & .73 \\
\hline 14. & Including the patient in planning his or her care & $4.03 \pm 1.00$ & .62 & .65 & & & .67 \\
\hline 16. & Returning to the patient voluntarily & $4.27 \pm 0.92$ & .71 & .70 & & & .74 \\
\hline 17. & Talking with the patient & $4.43 \pm 0.91$ & 63 & .65 & & & .67 \\
\hline 19. & Meeting the patient's stated and unstated needs & $4.16 \pm 0.91$ & 69 & .66 & & & .71 \\
\hline 22. & Showing concern for the patient & $4.58 \pm 0.84$ & .75 & .72 & & & .77 \\
\hline 9. & Knowing how to give shots, IVs, etc. & $4.89 \pm 0.93$ & .49 & & .59 & & .57 \\
\hline 10. & Being confident with the patient & $4.59 \pm 0.82$ & .66 & & .88 & & .88 \\
\hline 11. & Demonstrating professional knowledge and skill & $4.40 \pm 0.87$ & 69 & & .89 & & .91 \\
\hline 12. & Managing equipment skillfully & $4.37 \pm 0.87$ & .53 & & .79 & & .78 \\
\hline 18. & Encouraging the patient to call if there are problems & $4.90 \pm 0.93$ & .57 & & & .60 & .61 \\
\hline 20. & Responding quickly to the patient's call & $4.72 \pm 0.86$ & .62 & & & .72 & .72 \\
\hline 21. & Helping to reduce the patient's pain & $4.78 \pm 0.81$ & .67 & & & .86 & .84 \\
\hline 23. & $\begin{array}{l}\text { Giving the patient's treatments and medications } \\
\text { on time }\end{array}$ & $4.82 \pm 0.82$ & .57 & & & .76 & .75 \\
\hline \multirow[t]{2}{*}{24.} & Relieving the patient's symptoms & $4.62 \pm 0.80$ & .69 & & & .76 & .79 \\
\hline & Factors' descriptive statistics (M $\pm \mathrm{SD})$ & & & $4.24 \pm 0.66$ & $4.56 \pm 0.73$ & $4.77 \pm 0.67$ & \\
\hline
\end{tabular}




\section{3) Discriminant validity}

The discriminant validity $\left(\mathrm{r}^{2}\right)$ was adequate in the analysis of the 23-item CBI-K. The correlation matrix and square of the correlation between factors $i$ and $j$ are shown in Table 3. Results demonstrated that $\mathrm{AVE}_{1}(=.53)$ and $\mathrm{AVE}_{2}(=.68)$ were higher than the value for the square of the correlation between factor 1 and factor $2(=.45)$ and that $\mathrm{AVE}_{2}(=.68)$ and $\mathrm{AVE}_{3}(=.63)$ were higher than the value for the square of the correlation between factor 2 and factor $3(=.42)$.

\section{Reliability}

Reliability analysis was carried out on the CBI-K comprising 23 items. Cronbach's $\alpha$ coefficients for clinical nurses' responses to the CBI-K was. 95. Cronbach's $\alpha$ coefficient was .93 for factor 1 (empathy and supporting), .86 for factor 2 (knowledge and skill), and .85 for factor 3 (providing comfort)(Table 4). The mean inter-item correlation of the CBI-K was .43, with a range of .22 to .69 (not be presented a result table). Corrected item-total correlations were from .49 to .75 (Table 2).

\section{Description of the Korean Version of $\mathrm{CBI}$}

The CBI-K consists of 23 items on three factors: (a) Factor 1-empathy and supporting 14 items, (b) Factor 2-knowledge and skill, 4 items, (c) Factor 3-providing comfort, 5 items. The three factors presented a slightly different distribution of items from CBI-24[6]. All items of factor 3 (respectful) and factor 4 (connectedness), and three items $(16,17$, and 22) of factor 1 (assurance) of CBI-24 were loaded as one factor (empathy and supporting) in CBI-K.

Convergent validity both CBI-24 and CBI-K reported acceptable indices, but there are methodological differences in calculating convergent validity. The CBI-K that was drawn from this study using factor analysis showed a level of reliability as high as CBI-24 [6] (Table 4).

\section{DISCUSSION}

In this study, psychometric properties of the CBI-K for clinical nurses were investigated in Korea. In order to achieve this, content validity, construct validity, and reliability were verified.

The translation of the original text from English to Korean and vice versa were performed by qualified linguists. The instrument represented a high level of similarity with its original form following translation. To evaluate content validity indices, I-CVI should have an agreement of $80 \%$ or higher [23] and S-CVI/Ave should be $\geq 90 \%$ [30]. Results of this evaluation revealed that I-CVI was over $80 \%$

Table 3. Correlations, Discriminant and Convergent Validity of the CBI-K

$(N=408)$

\begin{tabular}{|c|c|c|c|c|c|c|c|}
\hline \multirow{2}{*}{ Variables } & \multicolumn{2}{|c|}{ Correlation matrix } & \multicolumn{3}{|c|}{$r^{2}$} & \multicolumn{2}{|c|}{ Convergent validity } \\
\hline & $\mathrm{F} 1$ & F2 & $r_{12}^{2}$ & $r_{13}{ }^{2}$ & $r_{23}{ }^{2}$ & AVE & $\mathrm{CR}$ \\
\hline $\mathrm{F} 1$ & - & & .45 & .61 & .42 & .53 & .94 \\
\hline $\mathrm{F} 2$ & .67 & - & & & & .68 & .89 \\
\hline F3 & .78 & .65 & & & & .63 & .90 \\
\hline
\end{tabular}

AVE=average variance extracted; $\mathrm{CR}=$ composite reliability.

F1: "Empathy and Supporting"; F2: "Knowledge and Skill"; F3: "Providing Comfort".

$r_{12}{ }^{2}$ : square of the correlation between factors 1 and $2 ; r_{13}{ }^{2}$ : square of the correlation between factors 1 and $3 ; r_{23}{ }^{2}:$ square of the correlation between factors 2 and 3 .

Table 4. Comparisons on Validity and Reliability between $\mathrm{CBI}-24$ and $\mathrm{CBI}-\mathrm{K}$

\begin{tabular}{|c|c|c|c|c|c|c|}
\hline \multirow{2}{*}{ Items } & \multirow{2}{*}{ Factors } & \multirow{2}{*}{ No. of items } & \multicolumn{3}{|c|}{ Convergent validity } & \multirow{2}{*}{ Reliability } \\
\hline & & & Pearson $\mathrm{r}^{\dagger}$ & AVE & $\mathrm{CR}$ & \\
\hline \multirow[t]{5}{*}{ CBI-24 [6] } & Overall & & .62 & & & .96 \\
\hline & Assurance & $16,17,18,20,21,22,23,24$ & & & & .92 \\
\hline & Knowledge and skill & $9,10,11,12,15$ & & & & .83 \\
\hline & Respectful & $1,3,5,6,13,19$ & & & & .92 \\
\hline & Connectedness & $2,4,7,8,14$ & & & & .87 \\
\hline \multirow[t]{4}{*}{ CBI-K } & Overall & & & & & .95 \\
\hline & Empathy and supporting & $1,2,3,4,5,6,7,8,13,14,16,17,19,22$ & & .53 & .94 & .93 \\
\hline & Knowledge and skill & $9,10,11,12$ & & .68 & .89 & .86 \\
\hline & Providing comfort & $18,20,21,23,24$ & & .63 & .90 & .85 \\
\hline
\end{tabular}

${ }^{\dagger}$ Pearson $\mathrm{r}$ was reported by calculating a correlation between the two instruments (CBI-24 and patient satisfaction) among patients. 
for all items and S-CVI/Ave was over 90\%, indicating good content validity.

Construct validity was examined based on factorial, convergent, and discriminant validity. Factorial validity was investigated via EFA and CFA. Three factors were derived for the CBI-K as a result of the EFA and CFA: 'empathy and supporting', 'knowledge and skill', and 'providing comfort'. Finfgeld-Connett [7] has described the structure of caring using terms 'intimate relationships', 'expert practice', and 'interpersonal sensitivity'. These structures overlapped with dimensions of caring behaviors in the present study. These three factors extracted in the EFA were not consistent with the previous study that reported four factors on CBI-24 [6]. There is a possibility that Korean clinical nurses might understand with different meanings for each item due to Korean cultural and situational context, unlike participants of the previous research. Most items loaded in the 'empathy and supporting' dimension (F1) - 14/23 (60.9\%) items. This dimension is the most exploratory one based on nurses' perspective of CBI-K. This might suggest that the factor of 'empathy and supporting' could be considered as the core meaning of nursing care in Korea.

Before discussing the model fit of CFA, we should consider the criteria of various model fit indices. In this study, the $x^{2}$ statistic showed statistically significant, meaning that there was a poor model fit. However, the $x^{2}$ statistic is very sensitive to sample size. It should not be the sole method used for deriving a conclusion about model fit [26]. For this reason, relative $x^{2}$, that is $x^{2} / \mathrm{df}$, is commonly used. When its value is less than 3.00 , the model fit is preferred. However, even when its values are between 3.00 and 4.00 or 5.00 , the model fit is assumed to be good [27]. Therefore, $x^{2} / \mathrm{df}$ value of $3.50 \mathrm{in}$ this sample indicated a good model fit. In the case of RMR and RMSEA, their values were less than .05 , indicating a good model fit. When RMSEA value is between .05 and .08 , the model fit is acceptable. When its value is less than .10, the model fit is moderate [26]. In the present study, values of RMR and RMSEA were .05 and .08 , respectively, indicating an acceptable model-fit. For GFI, AGFI, NFI, IFI. TLI, and CFI, if their values are equal to or greater than .90, it indicates a good fit of the model [26]. Although four of six indices in this study were slightly below the criteria, they were acceptable. Based on these indices, CFA provided an acceptable model fit, thus warranting factorial validity for the CBI-K.

Results of this study showed that factor loadings in all items were more than .50, with AVR values more than .50 and $C R$ values more than .70 . These values could be con- sidered as acceptable for convergent validity [28]. In other words, the analysis of convergent validity showed satisfactory results that highlighted capabilities of the CBI-K. In the previous study [6], convergent validity was verified with Pearson correlation between CBI-24 and the patient satisfaction scores. This study was verified by checking how well each items composed of one factor explains the factor to assess convergent validity and this results showed acceptable convergent validity as much as previous study.

For good discriminant validity, the AVE of one factor should be greater than the correlation coefficient between one factor and the other one. If any factor has smaller AVE than correlation coefficients, it means that these factors are correlated without measuring well-separated latent concepts [28]. In this study, the AVE of factor 1 was smaller than correlation coefficients between factor 1 (empathy and supporting) and factor 3 (providing comfort), meaning that factor 1 might be associated with factor 3 . Actually, factor 1 was a mixture of dimensions of "respectfulness", "assurance", and "connectedness" in a previous study [6]. This result may mean that latent factors in Korean specific-context could not be independent.

Results of this study showed that the CBI-K version demonstrated satisfactory reliability. The Cronbach's $\alpha$ coefficient of .95 indicates that the CBI-K is internally consistent, as in the English version [6]. Cronbach's $\alpha$ coefficients of all subscales of CBI-K version were above .70, confirming the stability of the subscales [29]. For the mean inter-item correlation in this study, all items met a minimum value of . 20 for item-total correlation [29]. Values of corrected item-total correlation were above .30, indicating that each item correlated with the total score, and this means that each item is measuring consistently something as a whole [29]. These findings support that CBI-K has good internal consistency.

\section{Study Limitations}

The nurses who participated in this study were from two hospitals located in a large city in Korea. Thus, our results might be different if nurses in other areas of Korea are recruited. There was no analysis of the criterion validity known to indicate how well the CBI-K version correlated with other well-established instruments (e.g., Caring Factor Survey [12]). The criterion-related validity should be tested in the future. Nevertheless, this study will contribute as the cornerstone of future research on caring behaviors from nurses' perspectives in clinical settings, which are increasingly attracting attention worldwide. In the future, it will be necessary to test the CBI-K in hospitals at 
other cities to determine whether the same factors will be extracted and whether their validity and reliability can be verified.

\section{CONCLUSION}

Findings of this study provide preliminary evidence that the 23-item CBI-K is a valid and reliable instrument for acquiring empirical knowledge on the caring behaviors of clinical nurses in Korea. These results suggest that CBI-K is an available instrument to measure caring behaviors from Korean nurses' perspective in the context of clinical nursing environment.

\section{CONFLICTS OF INTEREST}

The authors declared no conflict of interest.

\section{REFERENCES}

1. Watson J. Assessing and measuring caring in nursing and health science. 2nd ed. New York: Springer; 2008. p. 3-23.

2. Edvardsson D, Watt E, Pearce F. Patient experiences of caring and person-centeredness are associated with perceived nursing care quality. Journal of Advanced Nursing. 2017;73(1): 217-227. https://doi.org/10.1111/jan.13105

3. Fenizia E, Marchetti A, Biagioli V, Romano MC, Raso A, Gambera A, et al. Psychometric testing of the caring behaviors inventory for nursing students. Journal of Clinical Nursing. 2019;28(19-20):3567-3574. https://doi.org/10.1111/jocn.14950

4. Papastavrou E, Efstathiou G, Tsangari H, Suhonen R, LeinoKilpi H, Patiraki E, et al. A cross-cultural study of the concept of caring through behaviours: patients' and nurses' perspectives in six different EU countries. Journal of Advanced Nursing. 2012;68(5):1026-1037. https://doi.org/10.1111/j.1365-2648.2011.05807.x

5. Sarafis P, Rousaki E, Tsounis A, Malliarou M, Lahana L, Bamidis $\mathrm{P}$, et al. The impact of occupational stress on nurses' caring behaviors and their health related quality of life. BMC Nursing. 2016;15:56.

6. Wu Y, Larrabee JH, Putman HP. Caring behaviors inventory: a reduction of the 42-item instrument. Nursing Research. 2006; 55(1):18-25.

7. Finfgeld-Connett D. Meta-synthesis of caring in nursing. Journal of Clinical Nursing. 2008;17(2):196-204. https://doi.org/10.1111/j.1365-2702.2006.01824.x

8. Naidu A. Factors affecting patient satisfaction and healthcare quality. International Journal of Health Care Quality Assurance. 2009;22(4):366-381. https:// doi.org/10.1108/09526860910964834

9. Watson J. Nursing: human science and human care - a theory of nursing. Norwalk, Connecticut: Appleton-Century-Crofts; 1985. p. 1-111.

10. Swanson KM. Empirical development of a middle range theory of caring. Nursing Research. 1991;40(3):161-166.

https://doi.org/10.1097/00006199-199105000-00008

11. Watson J. Nursing: the philosophy and science of caring. Revised ed. Boulder, Colorado: University Press of Colorado; 2008. p. 19-44.

12. DiNapoli PP, Nelson J, Turkel M, Watson J. Measuring the caritas processes: caring factor survey. International Journal of Human Caring. 2010;14(3):15-20. https://doi.org/10.20467/1091-5710.14.3.15

13. Smith MC, Turkel MC, Wolf ZR. Caring in nursing classics: an essential resource. New York: Springer Publishing Company; 2013. p. 265-307.

14. Brown A, Kirpal S. Old Nurses with new qualifications are best: competing ideas about the skills that matter in nursing in Estonia, France, Germany and the UK. In: Warhurst C, Keep E, Grugulis I, editors. The skills that matter. basingstoke hampshire, Palgrave Macmillan; 2004. p. 225-241.

15. Larson PJ. Important nurse caring behaviors perceived by patients with cancer. Oncology Nursing Forum. 1984;11(6):46-50.

16. Wolf ZR. The caring concept and nurse identified caring behaviors. Topics in Clinical Nursing. 1986;8(2):84-93.

17. Wolf ZR, Giardino ER, Osborne PA, Ambrose MS. Dimensions of nurse caring. The Journal of Nursing Scholarship. 1994;26 (2):107-111. https://doi.org/10.1111/j.1547-5069.1994.tb00927.x

18. Flynn S. Who cares? A critical discussion of the value of caring from a patient and healthcare professional perspective. International Journal of Orthopaedic and Trauma Nursing. 2016;20: 28-39. https:// doi.org/10.1016/j.ijotn.2015.06.001

19. Karlou C, Papathanassoglou E, Patiraki E. Caring behaviours in cancer care in Greece. Comparison of patients', their caregivers' and nurses' perceptions. European Journal of Oncology Nursing. 2015;19(3):244-250.

https://doi.org/10.1016/j.ejon.2014.11.005

20. Sossong A, Poirier P. Patient and nurse perceptions of caring in rural United States. International Journal for Human Caring. 2013;17(1):79-85. https://doi.org/10.20467/1091-5710.17.1.79

21. Choi EH, Lee EN. Importance of nurses' caring behaviors as perceived by nurses and patients at emergency departments. The Journal of Korean Academic Society of Nursing Education. 2014;20(3):402-412. https://doi.org/10.5977/jkasne.2014.20.3.402

22. Kim YY, Lee BS. Importance of nurses's caring behaviors as perceived by staff nurses and patients. Journal of the Korean Academy of Fundamentals of Nursing. 1999;6(1):18-34.

23. Davis LL. Instrument review: getting the most from a panel of experts. Applied Nursing Research. 1992;5(4):194-197. 
https://doi.org/10.1016/S0897-1897(05)80008-4

24. Polit DF, Beck CT. The content validity index: are you sure you know what's being reported critique and recommendations. Research in Nursing \& Health. 2006;29(5):489-497. https://doi.org/10.1002/nur.20147

25. Tabachnick BG, Fidell LS. Using multivariate statistics. 5th ed. Boston: Pearson Education; 2007. 613 p.

26. Yu JP. Structural equation model's concept and understanding. Seoul: Hannarae publishing; 2012. p. 360-370.

27. Mueller RO. Basic principles of structural equation modeling: an introduction to LISREL and EQS. New York: Springer;
1996. p. 62-128.

28. Fornell C, Larcker DF. Evaluating structural equation models with unobservable variables and measurement error. Journal of Marketing Research. 1981;18(1):39-50.

https://doi.org/10.2307/3151312

29. Pallant J. SPSS survival manual: a step by step guide to data analysis using SPSS for windows. 3rd ed. New York: McGraw Hill Open University Press; 2007. p. 95-99.

30. Waltz CF, Strickland OL, Lenz ER. Measurement in nursing and health research. 3rd ed. New York: Springer; 2005. 178 p. 